3-1-1984

\title{
Relativistic Invariance and Zitterbewegung
}

James A. Lock

Cleveland State University, j.lock@csuohio.edu

Follow this and additional works at: https://engagedscholarship.csuohio.edu/sciphysics_facpub

Part of the Physics Commons

How does access to this work benefit you? Let us know!

\section{Publisher's Statement}

Copyright 1984 American Association of Physics Teachers. The article appeared in American Journal of Physics 52 (1984): 223-227 and may be found at http://aapt.scitation.org/doi/ $10.1119 / 1.13697$

\section{Original Citation}

Lock, James A. "Relativistic Invariance and Zitterbewegung." American Journal of Physics 52 (1984): 223-227.

\section{Repository Citation}

Lock, James A., "Relativistic Invariance and Zitterbewegung" (1984). Physics Faculty Publications. 66.

https://engagedscholarship.csuohio.edu/sciphysics_facpub/66

This Article is brought to you for free and open access by the Physics Department at EngagedScholarship@CSU. It has been accepted for inclusion in Physics Faculty Publications by an authorized administrator of

EngagedScholarship@CSU. For more information, please contact library.es@csuohio.edu. 


\title{
Relativistic invariance and Zitterbewegung
}

\author{
James A. Lock
}

\begin{abstract}
We examine the question of what it is about the structure of relativistic quantum mechanics that causes the nonintuitive phenomenon of the Zitterbewegung of particle position to exist. Further, we examine various assumptions concerning the measurement process which are implicit in the observation of Zitterbewegung.
\end{abstract}

\section{INTRODUCTION}

Over the last fifty years, the subject of Zitterbewegung of a free particle has been examined in many articles ${ }^{1-6}$ and standardly appears in textbooks on relativistic quantum mechanics. ${ }^{7-9}$ It is interpreted as a trembling of the position of the free particle superposed on its usual rectilinear motion. It is said to result from the fact that a particle wave packet cannot be localized to any size smaller than roughly its Compton wavelength ${ }^{10}$ without possessing both positive energy and negative energy Fourier components. When the expectation values of various functions of the position operator are calculated for such a wave packet state, the positive energy and negative energy Fourier components interfere producing either transient or steady-state vibrations in the expectation values with a frequency greater than $2 m c^{2} /$ $\hbar$.

In the present communication we consider a different aspect of the phenomenon of Zitterbewegung. Rather than being primarily concerned with the effects of Zitterbewegung, we address the more fundamental question of what it is about the structure of relativistic quantum mechanics that causes this nonintuitive phenomenon to occur in the first place. Secondly, we examine the assumptions implicit in the position measurement process which are necessary for the observation of Zitterbewegung.

One may claim that the answers to both of these questions concerning the origins of Zitterbewegung are not unknown. However, these questions of interpretation are important to the understanding of the phenemenon and they are usually at best only implicitly addressed in the standard treatments of the topic. Thus, we believe it to be of pedagogic value to explicitly address these questions in this communication. Our two conclusions appear at the end of Sec. III and at the beginning of Sec. IV. They are then repeated in Sec. V.

\section{RELATIVISTIC INVARIANCE AND FREE PARTICLE SYSTEMS}

In the quantum mechanics of a single particle, the particle position operator is associated with a dynamical property of the particle and provides a rule for the correspondence of measured values of that property with coordinates in three-dimensional space. In nonrelativistic quantum mechanics, the only correspondence deemed useful is the identity, i.e., that the position of a particle is its location in space. ${ }^{11}$ An example of this identity association is the assumed proportionality of the single particle charge density and the probability density in nonrelativistic quantum mechanics, $^{12.13}$

$$
\rho(\mathbf{r})=e|\psi(\mathbf{r})|^{2}
$$

By having a unitary operator $\hat{W}$ acting on each of the operators $\hat{O}$ and states $|\psi\rangle$ of the original system via the prescription

$$
|\psi\rangle^{\prime}=\hat{\boldsymbol{W}}|\psi\rangle
$$

and

$$
\hat{O}^{\prime}=\hat{W} \hat{O} \hat{W}^{-1} \text {, }
$$

it is always possible to generate an equivalent representation of the theory. In the transformation process, the physical interpretations of the various operators change but all matrix elements, and therefore all measurements, remain the same. Often, transforming to another representation is calculationally very useful such as when

$$
\hat{W}=\exp (i \hat{H} t)
$$

which connects the Schrödinger picture and Heisenberg picture versions of single particle quantum mechanics or when $\hat{W}$ is the Clebsch-Gordon matrix which connects the coupled and uncoupled versions of spin-orbital angular momentum space. In these two cases, however, the phys- 
ical meaning of the position operator $\hat{\mathbf{X}}$ remains the same. With other changes of representation the physical interpretation of the position operator may well change. Such is the case when, for example,

$$
\hat{W}=\exp \left[i(\hat{\mathbf{P}} \cdot \mathbf{a})^{2}\right]
$$

and

$$
\hat{\mathbf{X}}^{\prime}=\hat{\mathbf{X}}+2 \mathbf{a}(\hat{\mathbf{P}} \cdot \mathbf{a}) \text {. }
$$

In this situation, position in the new representation is a linear combination of position and momentum in the original representation. Such position-changing representations are deemed neither useful nor necessary in nonrelativistic quantum mechanics.

In relativistic quantum mechanics, a new situation arises with respect to similarity transformations of the theory. Correspondences for the position operator other than the identity association are meaningful and useful. ${ }^{14}$ The relativistic quantum mechanics of a single free particle of spin $S$, mass $m$, and which possesses both positive and negative energy states ${ }^{15}$ may be formulated in any one of an infinite number of equivalent reducible representations of the Poincaré group. One such representation, known as the canonical representation, ${ }^{16}$ assumes a form reminiscent of the Galilean quantum mechanics of a single free nonrelativistic particle. The canonical representation is expressed in terms of four basic particle dynamical variable operators, $\hat{\mathbf{X}}, \hat{\mathbf{P}}, \hat{\mathbf{\Sigma}}$, and $\hat{\Delta}$, which are descriptively termed the particle position operator, the particle momentum operator, the particle spin operator, and the particle energy sign operator, respectively. The time evolution of the free particle is expressed by the Schrödinger equation

$$
\hat{H}_{0}|\Psi\rangle_{t}=i \hbar \frac{\partial}{\partial t}|\Psi\rangle_{t},
$$

with the free particle Hamiltonian in this representation being

$$
\hat{H}_{0}=\hat{\Delta}\left(c^{2} \hat{\mathbf{P}} \cdot \hat{\mathbf{P}}+m^{2} c^{4} I\right)^{1 / 2} .
$$

The position operator

$$
\hat{\mathbf{X}}=i \hbar \nabla_{\mathbf{P}}
$$

has the same simple properties that the canonically conjugate position operator had in Galilean quantum mechanics. For example, let the the simultaneous eigenstates of the commuting operators $\{\hat{\mathbf{X}}, \hat{\mathbf{\Sigma}}, \hat{\Delta}\}$ be $|\mathbf{R}(0), A, \Delta\rangle_{0}$ at $t=0$ and let the simultaneous eigenstates of the commuting operators $\{\hat{\mathbf{P}}, \hat{\mathbf{\Sigma}}, \hat{\Delta}\}$ be $|\mathbf{P}, \Lambda, \Delta\rangle_{0}$ at $t=0$. The time evolution of these states is then

and

$$
\begin{aligned}
& |\mathbf{R}(0), \Lambda, \Delta\rangle_{t}=\exp \left(-i \hat{H}_{0} t\right)|\mathbf{R}(0), \Lambda, \Delta\rangle_{0} \\
& |\mathbf{P}, \Lambda, \Delta\rangle_{t}=\exp \left(-i \hat{H}_{0} t\right)|\mathbf{P}, \Lambda, \Delta\rangle_{0},
\end{aligned}
$$

and the localization property of the position eigenstates is

$$
\hat{\mathbf{X}}|\mathbf{R}(0), \Lambda, \Delta\rangle_{0}=\mathbf{R}|\mathbf{R}(0), \Delta, \Delta\rangle_{0} \text {. }
$$

Then, as in Galilean quantum mechanics, the position operator $\hat{\mathbf{X}}$ is a local operator with respect to the coordinate eignestates at the instant of localization

$$
{ }_{0}\left\langle\mathbf{R}^{\prime}(0), \Delta^{\prime}, \Delta^{\prime}|\hat{\mathbf{X}}| \mathbf{R}(0), \Lambda, \Delta\right\rangle_{0}=\mathbf{R}^{\prime} \delta\left(\mathbf{R}^{\prime}-\mathbf{R}\right) \delta_{\Lambda^{\prime}{ }_{\Lambda}} \delta_{\Delta^{\prime} \Delta} .
$$

In addition, the Fourier components of the $\delta$-function localized coordinate eigenstates have a single energy sign,

$$
\begin{aligned}
& \mid \mathbf{R}(0), \boldsymbol{A}, \Delta)_{t} \\
& \left.\quad=\int \frac{d^{3} P}{(2 \pi)^{3}} \exp (i \mathbf{P} \cdot \mathbf{R}) \exp (-i \Delta E t) \mid \mathbf{P}, \Lambda, \Delta\right)_{0} .
\end{aligned}
$$

Finally, the time evolution of wave-packet states possesses no Zitterbewegung. If the wave-packet state $|\Psi\rangle_{0}$ is

$$
|\Psi\rangle_{0}=\sum_{\Delta \Delta} \int \frac{d^{3} P}{(2 \pi)^{3}} A(\mathbf{P}, \Lambda, \Delta)|\mathbf{P}, \Lambda, \Delta\rangle_{0},
$$

then

$$
\begin{aligned}
& { }_{0}\left\langle\Psi\left|\exp \left(i \hat{H}_{0} t\right) \hat{\mathbf{X}} \exp \left(-i \hat{H}_{0} t\right)\right| \Psi\right\rangle_{0} \\
& =i \hbar \sum_{\Delta \Delta} \int \frac{d^{3} P}{(2 \pi)^{3}} A *(\mathbf{P}, A, \Delta)\left[\nabla_{\mathbf{P}} A(\mathbf{P}, A, \Delta)\right] \\
& \quad+c \sum_{\Delta \Delta} \int \frac{d^{3} P}{(2 \pi)^{3}}|A(\mathbf{P}, A, \Delta)|^{2} \frac{\Delta \mathbf{P}}{E} t \\
& \equiv \mathbf{R}_{0}+\mathbf{V}_{G} t .
\end{aligned}
$$

Other equivalent representations of the relativistic quan. tum mechanics of a single particle with both positive and negative energy states available may be obtained from the canonical representation by the similarity transformation with the unitary operator $\hat{W}(\hat{\mathbf{X}}, \hat{\mathbf{P}}, \hat{\mathbf{\Sigma}}, \hat{\Delta}) .{ }^{17,18}$ If the unitarily transformed representation is expressed in terms of the unitarily transformed operators

$$
\begin{aligned}
& \hat{\mathbf{x}}=\hat{W} \hat{\mathbf{X}} \hat{W}^{-1}, \\
& \hat{\mathbf{p}}=\hat{W} \hat{\mathbf{P}} \hat{W}^{-1}, \\
& \hat{\mathbf{\sigma}}=\hat{W} \hat{\mathbf{\Sigma}} \hat{W}^{-1},
\end{aligned}
$$

and

$$
\hat{\delta}=\hat{W} \hat{\Delta} \hat{W}^{-1}
$$

the Hamiltonian and all other operators in the transformed representation are the same functions of $\hat{\mathbf{x}}, \hat{\mathbf{p}}, \hat{\boldsymbol{\sigma}}$, and $\hat{\delta}$ as the original versions of the operators were of $\hat{\mathbf{X}}, \hat{\mathbf{P}}, \hat{\mathbf{\Sigma}}$, and $\hat{\Delta}$ in the original representation. But if the transformed representation operators are also expressed in terms of $\hat{\mathbf{X}}, \hat{\mathbf{P}}$, $\hat{\Sigma}$, and $\hat{\Delta}$, they assume very different functional forms and physical meanings than their counterparts did in the canonical representation. When the transformed representation is written in this second way, the position operator $\hat{\mathbf{X}}$ does not, in general, commute with the transformed energy sign operator $\hat{\delta}$. As a result, the matrix elements of $\hat{\mathbf{X}}$ taken with respect to superpositions of the eigenstates of the transformed Hamiltonian

$$
\hat{h}_{0}=\hat{W} \hat{H}_{0} \hat{W}^{-1}
$$

possess Zitterbewegung. Specifically, if $|\mathbf{p}, \lambda, \delta\rangle_{0}$ are the $\hat{h}_{0}$ is

$$
\hat{h}_{0}=\hat{\delta}\left(c^{2} \hat{\mathbf{p}} \cdot \hat{\mathbf{p}}+m^{2} c^{4} I\right)^{1 / 2},
$$

and $|\psi\rangle_{0}$ is the wave-packet state,

$$
|\psi\rangle_{0}=\sum_{\lambda \delta} \int \frac{d^{3} p}{(2 \pi)^{3}} a(\mathbf{p}, \lambda, \delta)|\mathbf{p}, \lambda, \delta\rangle_{0},
$$

then 


$$
\begin{aligned}
& { }_{0}\left\langle\psi\left|\exp \left(i \hat{h}_{0} t\right) \hat{\mathbf{X}} \exp \left(-i \hat{h}_{0} t\right)\right| \psi\right\rangle_{0} \\
& =i \hbar \sum_{\lambda \delta} \int \frac{d^{3} p}{(2 \pi)^{3}} a^{*}(\mathbf{p}, \lambda, \delta)\left[\nabla_{\mathbf{p}} a(\mathbf{p}, \lambda, \delta)\right] \\
& \quad+c \sum_{\lambda \delta} \int \frac{d^{3} p}{(2 \pi)^{3}}|a(\mathbf{p}, \lambda, \delta)|^{2} \frac{\delta \mathbf{p}}{E} t \\
& +i \hbar \sum_{\lambda \cdot \delta^{\prime} \delta \Delta \Delta} \int \frac{d^{3} p}{(2 \pi)^{3}} a^{*}\left(\mathbf{p}, \lambda^{\prime}, \delta^{\prime}\right) W_{\lambda^{\prime} \delta^{\prime} \wedge \Delta}(\mathbf{p}) \\
& \quad \times\left[\nabla_{\mathbf{p}} W_{\lambda \Delta \lambda \delta}(\mathbf{p})\right] a(\mathbf{p}, \lambda, \delta) \exp \left[-i\left(\delta^{\prime}-\delta\right) E t / \hbar\right]
\end{aligned}
$$

where

$$
E=\left(c^{2} p^{2}+m^{2} c^{4}\right)^{1 / 2},
$$

$W_{A \Delta \lambda \delta}(\mathbf{p})$ is the $2(2 S+1) \times 2(2 S+1)$ matrix realization of the operator $\hat{W}$, and the last term in Eq. (2.24) is the Zitterbewegung. Such position-changing transformed representations which are of great calculational value are the Dirac representation ${ }^{19}$ for $S=1 / 2$ and the Sakata-Taketani representation ${ }^{20}$ for $S=0$.

For a given $S$ and $m$, each equivalent representation provides an identical description of the relativistic quantum mechanics of a single free particle. With this being the case and without any further implicit assumptions, there is no physical reason to prefer one unitarily transformed representation over another for the description of the particle. Without further assumptions, one may simply avoid the problem of Zitterbewegung by appealing to the canonical representation with its basic position operator $\hat{\mathbf{X}}$. In this form of the theory, even though the particle has both energy signs available to it, the possibility of Zitterbewegung never arises.

\section{RELATIVISTIC INVARIANCE AND INTERACTING PARTICLE SYSTEMS}

In contrast to this, when considering the interaction of the particle with an external force center, since the external interaction must be expressed in a relativistically invariant way, the nature of the interaction often provides compelling calculational reasons to prefer one representation over another. For an external interaction of arbitrary complexity in the canonical representation, the procedure by which relativistic invariance is guaranteed or the restrictions on the interaction imposed by relativistic invariance are not known. ${ }^{21}$ However, if the interaction is unitarily transformable to a four-potential which is a local algebraic function of $\hat{X}$ and if the same transformed representation written in terms of $\hat{\mathbf{X}}, \hat{\mathbf{P}}, \Sigma$, and $\hat{\Delta}$ already gives rise to a manifestly covariant equation of motion, then the addition of the local four-vector interaction to the transformed representation via minimal coupling preserves the manifest covariance.

Actually, this procedure is usually carried out in the reverse order. ${ }^{17,18}$ For example, one begins with a manifestly covariant equation of motion with local four-vector interactions and minimal coupling such as the $S=1 / 2$ Dirac equation or the $S=0 \mathrm{~K}$ lein-Gordon equation. One then determines the form of the transformed representation tion vanist with these equations in the limit as the interacthe vanishes. Next the form of the operator $\hat{W}$ (known as the Foldy-Wouthuysen operator) connecting the transformed representation to the canonical representation is tetermined. Lastly one transforms the local four-vector interactions from the transformed representation to the ca- nonical representation via the similarity transformation with the Foldy-Wouthuysen operator $\hat{W}$. For example, let $|\mathbf{R}(0), \Lambda, \Delta\rangle_{0}$ be the simultaneous eigenstates of $\hat{\mathbf{X}}, \hat{\mathbf{\Sigma}}$, and $\hat{\Delta}$ at the instant of localization. Then the four-vector interaction $\hat{u}(\hat{\mathbf{X}})$ which is a local interaction in the transformed representation, i.e.,

$$
\begin{aligned}
& { }_{0}\left\langle\mathbf{R}^{\prime}(0), \Lambda^{\prime}, \Delta^{\prime}|\hat{u}(\hat{\mathbf{X}})| \mathbf{R}(0), \Lambda, \Delta\right\rangle_{0} \\
& =u_{A^{\prime}} \Delta^{\prime}{ }^{\prime} \Delta(\mathbf{R}) \delta\left(\mathbf{R}^{\prime}-\mathbf{R}\right)
\end{aligned}
$$

becomes the nonlocal interaction $\hat{U}$ in the canonical representation where

$$
\hat{U}=\hat{W}^{-1} \hat{u} \hat{W}
$$

and where its nonlocality is expressed by

$$
\begin{aligned}
& { }_{0}\left\langle\mathbf{R}^{\prime}(0), \Lambda^{\prime}, \Delta^{\prime}|\hat{U}| \mathbf{R}(0), \Lambda, \Delta\right\rangle_{0} \\
& =\sum_{\Delta \cdot \Delta \cdot \Delta^{-} \Delta^{-}} \int \frac{d^{3} p d^{3} p^{\prime}}{(2 \pi)^{6}} \int d^{3} R^{\prime \prime} \exp \left[\boldsymbol{i} \mathbf{P}^{\prime} \cdot\left(\mathbf{R}^{\prime}-\mathbf{R}^{\prime \prime}\right)\right] \\
& \times W_{\Delta \cdot \Delta \cdot A \cdot \Delta}^{-1} \cdot\left(\mathbf{P}^{\prime}\right) u_{A} \cdot \Delta_{\Delta} \cdot \Delta_{\Delta}-\left(\mathbf{R}^{\prime \prime}\right) \\
& \times W_{A-\Delta \sim \Delta}(\mathbf{P}) \exp [i \mathbf{P} \cdot(\mathbf{R} "-\mathbf{R})] \text {. }
\end{aligned}
$$

This is exactly what happens for the case of the electromagnetic interaction when the static electromagnetic fourpotential operators $[\hat{\mathbf{A}}(\hat{\mathbf{X}}), \hat{\boldsymbol{\Phi}}(\hat{\mathbf{X}})]$ are added to the free particle Dirac representation for $S=1 / 2$ via the minimal coupling prescription

$$
\hat{u}=e \hat{\Phi}(\hat{\mathbf{X}})-e \hat{\boldsymbol{\alpha}} \cdot \hat{\mathbf{A}}(\hat{\mathbf{X}}) \text {. }
$$

For the electromagnetic interaction in the transformed representation, the operator $\hat{\mathbf{X}}$ is interpreted as the electromagnetic position of the particle since from Eq. (3.1) a particle described by its localized eigenstate $|\mathbf{R}\rangle$ at the instant of localization experiences the electromagnetic potential at the single spatial coordinate $\mathbf{R}$. This procedure is not the only way in which to introduce external interactions into the theory in a relativistically invariant way. It is however the only way in which it is clear cut as to how to go about proving that the interactions so added are relativistically invariant. 22

It is a source of great relief and simplification that one does not have to go through such an elaborate procedure in order to guarantee the Galilean invariance of the theory of a single interacting nonrelativistic particle. For a free nonrelativistic particle, one begins with a representation of the Galilean group for which position is simple and for which the free Hamiltonian is

$$
\hat{H}_{0}=\hat{\mathbf{P}} \cdot \hat{\mathbf{P}} / 2 m \text {. }
$$

This is quite analogous to beginning the discussion of Poincaré invariance with the canonical representation where again the position operator had simple properties. For the case of local scalar or four-vector interactions, Galilean invariance is maintained by adding the interaction directly to the free particle Hamiltonian $\hat{H}_{0}$ in the original representation or by minimal coupling to the original representation free Hamiltonian respectively. One does not have to appeal to a different unitarily transformed representation in order to demonstrate the Galilean invariance of these external interactions. Galilean invariance allows position and interactions to possess simple properties in the same representation while relativistic invariance requires that position and interactions be simple in different representations. 


\section{ASSUMPTIONS IMPLICIT IN THE OBSERVATION OF ZITTERBEWEGUNG}

Since the Zitterbewegung arises in transformed representations of the Poincare group associated with manifestly covariant equations of motion and local four-vector interactions, its observation hinges on the local nature of the probing interaction of the measuring device used to measure position. Thus Zitterbewegung measurments give one information about the nature of the experimental probe as well as giving information about the particle being probed. An example of the dependence of the interpretation of one's observations on the assumptions one makes concerning the nature of interactions is provided by the Darwin term in the electromagnetic interaction of an electron bound in the Coulomb field of an atomic nucleus. One may take the point of view of the Dirac representation and claim that the Zitterbewegung of the electron's electromagnetic position in the local Coulomb field causes it to sample and time average the local electromagnetic potential over the range of the Zitterbewegung amplitude producing a

$$
\Delta E=\left(-e \hbar^{2} / 8 m^{2} c^{2}\right)\langle\psi|\nabla \cdot E| \psi\rangle
$$

shift in the bound state energy of the electron. Alternatively, one may take the point of view of the canonical representation and claim that the basic electron position operator possesses no Zitterbewegung but that the Coulomb interaction with the atomic nucleus is nonlocal when expressed in terms of this position operator. ${ }^{23}$ Then due to the nonlocality of the electromagnetic interaction, the electron at a given point location experiences the interaction at that coordinate averaged with the interaction strength evaluated over a small neighborhood of spatial coordinates near to it. The same energy shift results from this alternative point of view. The Darwin interaction is a reflection of the inability of particle position and electrostatic interactions to be simultaneously described in a simple way in a given representation. One may interpret the effect to be due to either of the two complementary causes with equal validity.

The same interpretive assumptions are inherent in the terminology "the Zitterbewegung of a free particle." The terminology describes the fuzziness in position measurements that one would observe if the free particle under consideration was being continuously sampled by an arbitrarily weak probe, and if one were monitoring the observable quantity in terms of which the probing potential was a local four-vector. In terms of the canonical representation, this same fuzzyness in position measurements would be interpreted as being due to the nonlocal character of the probing interaction in that representation. Both for the Darwin interaction and for the free particle example, we prefer to think of the nonintuitive effects as being due to position Zitterbewegung rather than to force nonlocality. This is because, motivated by the way we parameterize fields in classical physics, we are more comfortable in preferring to think of common interactions such as the Coulomb force as being local interactions.

It is less clear cut as to what options of interpretation are useful if one considers the case where the probing interaction may well be intrinsically nonlocal, i.e., be a nonlocal function of $\hat{\mathbf{X}}$ in any unitarily transformed representa-
tion. ${ }^{22}$ In this case, the probing interaction special simplicity in any transformed representatios on no one may with complete validity, describesentation. Then system in the canonical representation where if nothing else, the position operator $\hat{\mathbf{X}}$ is simple. In the canonical representation, the Zitterbewegung of $\hat{\mathbf{X}}$ cannot occur. As. suming one knows how to experimentally measure $\hat{\mathbf{X}}$ in the canonical representation, any trembling in experimental observations of position would be due to the intrinsic non. locality of the probing interaction.

\section{SUMMARY}

The requirement that relativistic quantum mechanical theories allow both positive and negative energy states is a necessary but not sufficient condition for the existence of Zitterbewegung. From classical physics and nonrelativistic quantum mechanics, we carry over two intuitive hopes for relativistic quantum mechanics, that in some general way position possesses simple properties and that in some general way interactions possess simple properties. Crucial to the existence of Zitterbewegung is the fact that, as seen in Sec. III, relativistic invariance demands that both of these intuitive hopes cannot be simultaneously fulfilled. One has the option of whether to allow position to be simple or to allow interactions to be simple. But one can never take both options at once. If the particle position operator maintains all the intuitive properties which it possessed in Galilean quantum mechanics, then interactions are forced to be nonlocal functions of that position. That is, the observed strength of the interaction is its value at the spatial coordinate where the particle is located, averaged with the interaction strength at neighboring spatial coordinates where the particle is not located. On the other hand, if one expresses interactions as algebraic functions of a local position operator, then that position which the interactions are local in possess Zitterbewegung. Also, since Zitterbewegung is a consequence of the assumption of the local character of the probing interaction, if one is not going to have any local four-vector interactions present, one is not going to have any Zitterbewegung present either.

'G. Briet, Proc. Natl. Acad. Sci. 14, 553 (1928); 17, 70 (1931).

${ }^{2}$ E. Schrödinger, Zit. Pruess. Akad. 24, 418 (1930).

${ }^{3}$ K. Huang, Am. J. Phys. 20, 479 (1952).

${ }^{4}$ R. F. Guertin and E. Guth, Phys. Rev. D 7, 1057 (1973).

${ }^{5}$ J. A. Lock, Am. J. Phys. 47, 797 (1979).

${ }^{6}$ M. G. Fuda and E. Furlani, Am. J. Phys. 50, 545 (1982).

${ }^{7}$ E. Merzbacher, Quantum Mechanics (Wiley, New York, 1970), Chap. 24, Sec. 2.

${ }^{8}$ A. Messiah, Quantum Mechanics (North Holland, Amsterdam, 1966), Chap. XX, Secs. 35,37

${ }^{9}$ J. J. Sakurai, Advanced Quantum Mechanics (Addison-Wesley, Reading, MA, 1967), Chap. 3, Secs. 6 and 7.

${ }^{10}$ T. D. Newton and E. P. Wigner, Rev. Mod. Phys. 21, 400 (1949).

'This is reinforced by the fact that in nonrelativistic quantum mechanics, the particle position operator and spatial coordinates transform in the same manner under Galilean changes of reference frame. In relativistic quantum mechanics, position operators and spatial coordinates transform in different manners under Lorentz changes of reference frame.

See, for example, J. A. Lock, Found. Phys. 12, 743 (1982).

${ }^{12}$ M. E. Rose, Phys. Rev. 73, 279 (1948).

${ }^{13}$ B. Hahn, D. G. Ravenhall, and R. Hofstadter, Phys. Rev. 101, 1131 (1956).

${ }^{14}$ Different correspondences for the position operator in the same representation are identical to employing the same operator for position in different representations. We adopt this second point of view. So rather than treating the mean position operator $\hat{\mathbf{X}}$ in the Dirac representation [Eq. (2.17)], we consider it as the position operator $\hat{\boldsymbol{X}}$ in the canonical representation.

${ }^{15} \mathrm{~A}$ single free particle with only a single energy sign is described by an 
irreducible representation of the Poincare group and does not have the ibility of Zitterbewegung in any unitarily transformed version of the theory.

Idy, Phys. Rev. 102, 568 (1956)

${ }^{16}$ L. L. Foldy, Phys. Rev. Wouthuysen, Phys. Rev. 78, 29 (1950).

"L. L. Foldy and S. A. Willars, Rev. Mod. Phys. 30, 24 (1958).

"H. Feshbach and F. Will. R. Soc. London, Ser. A 117, 610 (1928).

IDP. A. M. Dirac, Prokentani, Sci. Pap. Inst. Phys. Chem. Res. Tokyo 38,

zas. Sakata and M. Taken reprinted as Prog. Theor. Phys. Suppl. No. 1, 84 $1(19$

(1955).
${ }^{2}$ For the related system of two particles of a single energy sign interacting via internal forces, the restrictions imposed by relativistic invariance are known in principle. See for example B. Bakamjian and L. H. Thomas, Phys. Rev. 92, 1300 (1953); and L. L. Foldy, ibid. 122, 275 (1961).

${ }^{22} \mathrm{It}$ is not known whether all of the fundamental interactions in nature may be written as local vectors. Whether or not this turns out to be true, it still may be the case that useful phenomenological approximations to them may not be able to be written as local four-vectors.

${ }^{23}$ In the canonical representation, the Coulomb interaction is an algebraic function of the position $\hat{W}^{-1} \hat{\mathbf{X}} \hat{W}$ which is nonlocal in the eigenstates of $\hat{\mathbf{X}}$. Thus the Coulomb interaction is nonlocal in the eigenstates of $\hat{\mathbf{X}}$. This point of view is emphasized in Ref. 18.

Post-print standardized by MSL Academic Endeavors, the imprint of the Michael Schwartz Library at Cleveland State University, 2017. 